\title{
NON-MEAGER FREE SETS FOR MEAGER RELATIONS ON POLISH SPACES
}

\author{
TARAS BANAKH, LYUBOMYR ZDOMSKYY
}

\begin{abstract}
We prove that for each meager relation $E \subset X \times X$ on a Polish space $X$ there is a nowhere meager subspace $F \subset X$ which is $E$-free in the sense that $(x, y) \notin E$ for any distinct points $x, y \in F$.
\end{abstract}

\section{INTRODUCTION}

This paper is devoted to the problem of finding non-meager free subsets for meager relations on Polish spaces. For a relation $E \subset X \times X$, a subset $F \subset X$ is called $E$-free if $(x, y) \notin E$ for any distinct points $x, y \in F$. This is equivalent to saying that $F^{2} \cap E \subset \Delta_{X}$ where $\Delta_{X}=\left\{(x, y) \in X^{2}: x=y\right\}$ is the diagonal of $X^{2}$.

The problem of finding "large" free sets for certain "small" relations was considered by many authors, see [10], 11], 9], 6], 7]. Observe that the classical Mycielski-Kuratowski Theorem [8, 18.1] implies that for each meager relation $E \subset X^{2}$ on a perfect Polish space $X$ there is an $E$-free perfect subset $F \subset X$. We recall that a subset of a Polish space is perfect if it is closed and has no isolated points. Nonetheless the following result seems to be new.

Theorem 1. For each meager relation $E \subset X^{2}$ on a Polish space $X$ there is an $E$-free nowhere meager subspace $B \subset X$. Moreover, if the set of isolated points is not dense in $X$ then $B$ may be chosen of any cardinality $\kappa \in[\operatorname{cof}(\mathcal{M}), \mathfrak{c}]$.

Let us recall that a subspace $A$ of a topological space $X$

- is meager in $X$, if $A$ can be written as a countable union $A=\bigcup_{n \in \omega} A_{n}$ of nowhere dense subsets of $X$;

- is nowhere meager in $X$, if for any non-empty open set $U \subset X$ the intersection $U \cap A$ is not meager in $X$.

It is clear that a subset $A \subset X$ of a Polish space $X$ is nowhere meager if and only if $A$ is dense in $X$ and contains no open meager subspace. By definition, $\operatorname{cof}(\mathcal{M})$ is the minimal cardinality of a collection $\mathcal{X}$ of meager subsets of the Baire space $\omega^{\omega}$ such that for every meager $A \subset \omega^{\omega}$ there exists $X \in \mathcal{X}$ containing $A$. It is known [5] that $\operatorname{cof}(\mathcal{M})=\mathfrak{c}$ under Martin's Axiom, and $\operatorname{cof}(\mathcal{M})<\mathfrak{c}$ in some models of ZFC, see [4].

Theorem 1 will be proved in Section 3. One of its applications is the existence of a first-countable uniform Eberlein compact space which is not supercompact (see [1, 5.2]), which was our initial motivation for considering free non-meager sets for meager relations. The following simple example shows that the nowhere meager set $F$ in Theorem 1 cannot have the Baire property. We recall that a subset $A$ of a topological space $X$ has the Baire property in $X$ if for some open set $U \subset X$ the symmetric difference $A \triangle U=(A \backslash U) \cup(U \backslash A)$ is meager in $X$.

Example 2. For the nowhere dense relation

$$
E=\bigcup_{n \in \omega}\left\{(x, y) \in \mathbb{R}^{2}:|x-y|=2^{-n}\right\} \subset \mathbb{R} \times \mathbb{R}
$$

on the real line $\mathbb{R}$, each $E$-free subset $F \subset \mathbb{R}$ with the Baire property is meager.

2010 Mathematics Subject Classification. Primary: 54E52, 54E50. Secondary: 54D80.

Key words and phrases. meager relation, free set.

The first author has been partially financed by NCN grant DEC-2011/01/B/ST1/01439. The second author is a recipient of an APART-fellowship of the Austrian Academy of Sciences. 
Proof. Assuming that $F$ is not meager, and using the Baire property of $F$, find a non-empty open subset $U \subset \mathbb{R}$ such that $U \triangle F$ is meager and hence lies in some meager $F_{\sigma}$-set $M \subset \mathbb{R}$. Then $G=U \backslash M \subset F$ is a dense $G_{\delta}$-set in $U$. By the Steinhaus-Pettis Theorem [8, 9.9], the difference $G-G=\{x-y: x, y \in G\}$ is a neighborhood of zero in $\mathbb{R}$ and hence $2^{-n} \in G-G$ for some $n \in \omega$. Then any points $x, y \in G \subset F$ with $|x-y|=2^{-n}$ witness that the set $F \ni x, y$ is not $E$-free.

Remark 3. By a classical result of Solovay [12], there are models of ZF in which all subsets of the real line have the Baire property. In such models each $E$-free subset for the relation $E=\bigcup_{n \in \omega}\{(x, y) \in$ $\left.\mathbb{R}^{2}:|x-y|=2^{-n}\right\}$ is meager. This means that the proof of Theorem 1 must essentially use the Axiom of Choice.

\section{Some AuXiliary Results}

We recall [2] that a family $\mathcal{F}$ of infinite subsets of a countable set $X$ is called a semifilter, if $A \in \mathcal{F}$ provided $F \subset^{*} A \subset X$ for some set $F \in \mathcal{F}$. Here $F \subset^{*} A$ means that $F \backslash A$ is finite. Each semifilter on $X$ is contained in the semifilter $[X]^{\omega}$ of all infinite subsets of $X$. The semifilter $[X]^{\omega}$ is a subset of the power set $\mathcal{P}(X)$ which can be identified with the Tychonoff product $2^{X}$ via characteristic functions. So, we can speak about topological properties of semifilters as subspaces of the compact Hausdorff space $\mathcal{P}(X)$. According to Talagrand's characterization of meager semifilters on $\omega$, a semifilter $\mathcal{F}$ on a countable set $X$ is meager (as a subset of $\mathcal{P}(X)$ ) if and only if $\mathcal{F}$ can be enlarged to a $\sigma$-compact semifilter $\tilde{\mathcal{F}} \subset[X]^{\omega}$. This characterization implies the following:

Corollary 4. For any finite-to-one map $\phi: X \rightarrow Y$ between countable sets, a semifilter $\mathcal{F} \subset \mathcal{P}(X)$ is meager if and only if the semifilter $\phi[\mathcal{F}]=\left\{E \subset Y: \phi^{-1}(E) \in \mathcal{F}\right\} \subset \mathcal{P}(Y)$ is meager.

We recall that a map $f: X \rightarrow Y$ between two sets is called finite-to-one if for each $y \in Y$ the preimage $\psi^{-1}(y)$ is finite and non-empty. In particular, each monotone surjection $\psi: \omega \rightarrow \omega$ is finite-to-one.

A key ingredient of the proof of Theorem 1 in the following proposition.

Proposition 5. For any meager relation $E \subset 2^{\omega} \times 2^{\omega}$ on the Cantor cube $2^{\omega}$ there is a family $\left(G_{\alpha}\right)_{\alpha<\mathfrak{c}}$ of nowhere meager subsets in $2^{\omega}$ such that $\left(G_{\alpha} \times G_{\beta}\right) \cap E=\emptyset$ for any distinct ordinals $\alpha, \beta<\mathfrak{c}$.

Proof. Using the fact that the points of the Cantor cube $2^{\omega}$ can be identified with the branches of the binary tree $2^{<\omega}=\bigcup_{n \in \omega} 2^{n}$, we can find a closed subset $\left\{A_{\alpha}\right\}_{\alpha<\mathfrak{c}}$ of $\mathcal{P}(\omega)=2^{\omega}$ which consists of infinite subsets of $\omega$ and is almost disjoint in the sense that $A_{\alpha} \cap A_{\beta}$ is finite for any distinct ordinals $\alpha, \beta<\mathfrak{c}$. The compactness of $\left\{A_{\alpha}\right\}_{\alpha<\mathfrak{c}}$ in $2^{\omega}$ implies the existence of a monotone surjection $\varphi: \omega \rightarrow \omega$ such that $\varphi\left(A_{\alpha}\right)=\omega$ for all $\alpha<\mathfrak{c}$.

Fix any free ultrafilter $\mathcal{U}$ on $\omega$ and for every $\alpha<\mathfrak{c}$ choose an ultrafilter $\mathcal{U}_{\alpha}$ on $\omega$ extending the family $\left\{A_{\alpha} \cap \varphi^{-1}[U]: U \in \mathcal{U}\right\}$. The almost disjoint property of the family $\left\{A_{\alpha}\right\}_{\alpha<\mathfrak{c}}$ guarantees that $\omega \backslash A_{\alpha} \in \mathcal{U}_{\xi}$ for any distinct ordinals $\alpha, \xi<\mathfrak{c}$.

Lemma 6. For every $\alpha<\mathfrak{c}$, the filter

$$
\mathcal{F}_{\alpha}=\mathcal{P}\left(\omega \backslash A_{\alpha}\right) \cap \bigcap_{\alpha \neq \xi<\mathfrak{c}} \mathcal{U}_{\xi}
$$

is non-meager in $\mathcal{P}\left(\omega \backslash A_{\alpha}\right)$.

Proof. By Corollary 4, the filter $\mathcal{F}_{\alpha}$ is not meager in $\mathcal{P}\left(\omega \backslash A_{\alpha}\right)$ as its image $\varphi\left[\mathcal{F}_{\alpha}\right]=\{E \subset \omega$ : $\left.\varphi^{-1}[E] \in \mathcal{F}_{\alpha}\right\}$ coincides with the ultrafilter $\mathcal{U}$ and hence is not meager in $\mathcal{P}(\omega)$.

Let $E \subset 2^{\omega} \times 2^{\omega}$ be a meager relation on $2^{\omega}$. By [3, Theorem 2.2.4], there exist a monotone surjection $\phi: \omega \rightarrow \omega$ and functions $f_{0}, f_{1}: \omega \rightarrow 2$ such that

$$
E \subset\left\{\left(g, g^{\prime}\right) \in 2^{\omega} \times 2^{\omega}: \forall^{\infty} n \in \omega\left(g \left\lceil\phi^{-1}(n) \neq f_{0}\left\lceil\phi^{-1}(n)\right) \vee\left(g^{\prime}\left\lceil\phi^{-1}(n) \neq f_{1}\left\lceil\phi^{-1}(n)\right)\right\} .\right.\right.\right.\right.
$$


For every ordinal $\alpha<\mathfrak{c}$ consider the subset

$$
\begin{aligned}
G_{\alpha}=\left\{g \in 2^{\omega}:\right. & \exists X_{0}, X_{1} \in \mathcal{U}_{\alpha} \backslash \bigcup_{\alpha \neq \xi<\mathfrak{c}} \mathcal{U}_{\xi} \\
& \left(X_{0} \subset X_{1}\right) \wedge\left(g \left\lceil\phi^{-1}\left[X_{0}\right]=f_{0}\left\lceil\phi^{-1}\left[X_{0}\right]\right) \wedge\left(g\left\lceil\phi^{-1}\left[\omega \backslash X_{1}\right]=f_{1}\left\lceil\phi^{-1}\left[\omega \backslash X_{1}\right]\right)\right\}\right.\right.\right.
\end{aligned}
$$

in the Cantor cube $2^{\omega}$.

Lemma 7. For every ordinal $\alpha<\mathfrak{c}$ the set $G_{\alpha}$ is nowhere meager in $2^{\omega}$.

Proof. Since $G_{\alpha}$ is closed under finite modifications of its elements, it is enough to show that $G_{\alpha}$ is non-meager in $2^{\omega}$. Observe that $G_{\alpha}$ contains the set

$$
\begin{aligned}
& G_{\alpha}^{\prime}=\left\{g \in 2^{\omega}:\right. \exists Y_{0} \in \mathcal{U}_{\alpha} \cap \mathcal{P}\left(A_{\alpha}\right) \exists Y_{1} \in \mathcal{P}\left(\omega \backslash A_{\alpha}\right) \backslash \bigcup_{\alpha \neq \xi<\mathfrak{c}} \mathcal{U}_{\xi} \\
&\left(g \left\lceil\phi^{-1}\left[Y_{0}\right]=f_{0}\left\lceil\phi^{-1}\left[Y_{0}\right]\right) \wedge\left(g\left\lceil\phi^{-1}\left[\omega \backslash\left(A_{\alpha} \cup Y_{1}\right)\right]=f_{1}\left\lceil\phi^{-1}\left[\omega \backslash\left(A_{\alpha} \cup Y_{1}\right)\right]\right)\right\} .\right.\right.\right.
\end{aligned}
$$

Indeed, if $g \in G_{\alpha}^{\prime}$ is witnessed by $Y_{0}, Y_{1}$, then $X_{0}=Y_{0}$ and $X_{1}=A_{\alpha} \cup Y_{1}$ are witnessing that $g \in G_{\alpha}$. Now $G_{\alpha}^{\prime}$ may be written as the product $R_{\alpha} \times H_{\alpha}$, where

$$
R_{\alpha}=\left\{g \in 2^{\phi^{-1}\left[A_{\alpha}\right]}: \exists Y_{0} \in \mathcal{U}_{\alpha} \cap \mathcal{P}\left(A_{\alpha}\right) \quad\left(g\left\lceil\phi^{-1}\left[Y_{0}\right]=f_{0}\left\lceil\phi^{-1}\left[Y_{0}\right]\right)\right\}\right.\right.
$$

and

$$
\begin{aligned}
H_{\alpha}=\left\{g \in 2^{\phi^{-1}\left[\omega \backslash A_{\alpha}\right]:}:\right. & \exists Y_{1} \in \mathcal{P}\left(\omega \backslash A_{\alpha}\right) \backslash \bigcup_{\alpha \neq \xi<\mathfrak{c}} \mathcal{U}_{\xi} \\
& \left(g\left\lceil\phi^{-1}\left[\omega \backslash\left(A_{\alpha} \cup Y_{1}\right)\right]=f_{1}\left\lceil\phi^{-1}\left[\omega \backslash\left(A_{\alpha} \cup Y_{1}\right)\right]\right)\right\} .\right.
\end{aligned}
$$

Thus it suffices to show that both $R_{\alpha}$ and $H_{\alpha}$ are non-meager. By the homogeneity of $2^{\omega}$ there is no loss of generality to assume that $f_{0}\left\lceil\phi^{-1}\left[A_{\alpha}\right] \equiv 1\right.$ and $f_{1}\left\lceil\phi^{-1}\left[\omega \backslash A_{\alpha}\right] \equiv 1\right.$.

With $f_{1}$ as above we see that $H_{\alpha}$ is simply the set of characteristic functions of elements of the semifilter

$$
\mathcal{H}_{\alpha}=\left\{Z \subset \phi^{-1}\left[\omega \backslash A_{\alpha}\right]: \exists Y_{1} \in \mathcal{P}\left(\omega \backslash A_{\alpha}\right) \backslash \bigcup_{\alpha \neq \xi<\mathfrak{c}} \mathcal{U}_{\xi} \quad\left(\phi^{-1}\left[\omega \backslash\left(A_{\alpha} \cup Y_{1}\right)\right] \subset Z\right)\right\}
$$

on $\phi^{-1}\left[\omega \backslash A_{\alpha}\right]$. Therefore

$$
\phi\left[\mathcal{H}_{\alpha}\right]=\left\{T \subset \omega \backslash A_{\alpha}: \exists Y_{1} \in \mathcal{P}\left(\omega \backslash A_{\alpha}\right) \backslash \bigcup_{\alpha \neq \xi<\mathfrak{c}} \mathcal{U}_{\xi} \quad\left(\omega \backslash\left(A_{\alpha} \cup Y_{1}\right) \subset T\right)\right\} .
$$

Observe that $Y_{1} \in \mathcal{P}\left(\omega \backslash A_{\alpha}\right) \backslash \bigcup_{\alpha \neq \xi<\mathfrak{c}} \mathcal{U}_{\xi}$ iff $\omega \backslash\left(A_{\alpha} \cup Y_{1}\right) \in \bigcap_{\alpha \neq \xi<\mathfrak{c}} \mathcal{U}_{\xi}$, and hence $\phi\left[\mathcal{H}_{\alpha}\right]$ is equal to the filter $\mathcal{P}\left(\omega \backslash A_{\alpha}\right) \cap \bigcap_{\alpha \neq \xi<\mathfrak{c}} \mathcal{U}_{\xi}$ which is non-meager in $\mathcal{P}\left(\omega \backslash A_{\alpha}\right)$ by Lemma 6 , and consequently the filter $\mathcal{H}_{\alpha}$ is non-meager in $\mathcal{P}\left(\phi^{-1}\left[\omega \backslash A_{\alpha}\right]\right)$ by Corollary 4 . In other words, $H_{\alpha}$ is a non-meager subset of $2^{\phi^{-1}\left[\omega \backslash A_{\alpha}\right]}$.

The proof of the fact that $R_{\alpha}$ is non-meager is analogous. However, we present it for the sake of completeness. With $f_{0}$ as above we see that $R_{\alpha}$ is simply the set of characteristic functions of elements of the semifilter

$$
\mathcal{R}_{\alpha}=\left\{Z \subset \phi^{-1}\left[A_{\alpha}\right]: \exists Y_{0} \in \mathcal{P}\left(A_{\alpha}\right) \cap \mathcal{U}_{\alpha}\left(\phi^{-1}\left[Y_{0}\right] \subset Z\right)\right\}
$$

on $\phi^{-1}\left[A_{\alpha}\right]$. It follows that

$$
\phi\left[\mathcal{R}_{\alpha}\right]=\left\{T \subset A_{\alpha}: \exists Y_{0} \in \mathcal{P}\left(A_{\alpha}\right) \cap \mathcal{U}_{\alpha}\left(Y_{0} \subset T\right)\right\}=\mathcal{P}\left(A_{\alpha}\right) \cap \mathcal{U}_{\alpha}
$$

is a non-meager ultrafilter on $A_{\alpha}$ and then $\mathcal{R}_{\alpha}$ is a non-meager semifilter on $\phi^{-1}\left[A_{\alpha}\right]$ according to Corollary 4. Consequently, $R_{\alpha}$ is a non-meager subset of $2^{\phi^{-1}\left[A_{\alpha}\right]}$.

Lemma 8. For any distinct ordinals $\alpha, \beta<\mathfrak{c}$ we get $\left(G_{\alpha} \times G_{\beta}\right) \cap E=\emptyset$. 
Proof. Assume conversely that $\left(G_{\alpha} \times G_{\beta}\right) \cap E$ contains some pair $\left(g_{\alpha}, g_{\beta}\right)$. Fix sets $X_{0}^{\alpha}, X_{1}^{\alpha}$ and $X_{0}^{\beta}, X_{1}^{\beta}$ witnessing that $g_{\alpha} \in G_{\alpha}$ and $g_{\beta} \in G_{\beta}$, respectively. The intersection $X_{0}^{\alpha} \cap\left(\omega \backslash X_{1}^{\beta}\right)$ is infinite: otherwise $X_{0}^{\alpha} \subset^{*} X_{1}^{\beta}$ and $X_{1}^{\beta} \in \mathcal{U}_{\alpha}$, which contradicts the definition of $G_{\beta}$. Thus the set $X_{0}^{\alpha} \backslash X_{1}^{\beta}$ is infinite and for every $n \in X_{0}^{\alpha} \backslash X_{1}^{\beta}$ we get $g_{\alpha}\left\lceil\phi^{-1}(n)=f_{0}\left\lceil\phi^{-1}(n)\right.\right.$ and $g_{\beta}\left\lceil\phi^{-1}(n)=f_{1}\left\lceil\phi^{-1}(n)\right.\right.$, which implies $\left(g_{\alpha}, g_{\beta}\right) \notin E$.

This completes the proof of Proposition 5.

Using the well-known fact that each perfect Polish space $X$ contains a dense $G_{\delta}$-subset homeomorphic to the space of irrationals $\omega^{\omega}$, we can generalize Proposition 5 as follows.

Proposition 9. For any meager relation $E \subset X \times X$ on a perfect Polish space $X$ there is a family $\left(G_{\alpha}\right)_{\alpha<\mathfrak{c}}$ of nowhere meager subsets in $X$ such that $\left(G_{\alpha} \times G_{\beta}\right) \cap E=\emptyset$ for any distinct ordinals $\alpha, \beta<\mathfrak{c}$.

\section{Proof of Theorem 1}

Let $E \subset X \times X$ be a meager relation on a Polish space $X$. If the set $D$ of isolated points is dense in $X$, then $B=D$ is a required nowhere meager $E$-free subset of $X$. So, we assume that the set $D$ is not dense in $X$. Then the open subspace $Y=X \backslash \bar{D}$ of $X$ is not empty and has no isolated points. Let $\kappa \in[\operatorname{cof}(\mathcal{M}), \mathfrak{c}]$ be any cardinal. By Proposition 9, there is a family $\left(G_{\alpha}\right)_{\alpha<\kappa}$ of nowhere meager subsets in $Y$ such that $\left(G_{\alpha} \times G_{\beta}\right) \cap E=\emptyset$ for any distinct ordinals $\alpha, \beta<\kappa$.

Let $\mathcal{U}$ be a countable base of the topology of $Y$ and $\mathcal{X}$ be a cofinal with respect to inclusion family of meager subsets in $Y$ of size $\kappa$. It is clear that the set $\mathcal{U} \times \mathcal{X}$ has cardinality $\kappa$ and hence can be enumerated as $\mathcal{U} \times \mathcal{X}=\left\{\left(U_{\alpha}, X_{\alpha}\right): \alpha<\kappa\right\}$. Since the set $D$ is at most countable and $E$ is meager in $X \times X$, the set $E_{0}=\{y \in Y: \exists x \in D \quad(x, y) \in E$ or $(y, x) \in E\}$ is meager in $Y$. For every ordinal $\alpha<\kappa$ the set $G_{\alpha}$ is nowhere meager in $Y$, which allows us to find a point $y_{\alpha} \in U_{\alpha} \cap G_{\alpha} \backslash\left(X_{\alpha} \cup E_{0}\right)$. Then $B=D \cup\left\{y_{\alpha}\right\}_{\alpha<\kappa}$ is a nowhere meager $E$-free set in $X$.

\section{REFERENCES}

[1] T. Banakh, Z. Kosztołowicz, S. Turek, Hereditarily supercompact spaces, preprint (http://arxiv.org/abs/1301.5297).

[2] T. Banakh, L. Zdomskyy, Coherence of Semifilters: a survey, in: Selection Principles and Covering Properties in Topology (L. Kocinac ed.), Quaderni di Matematica. 18 (2006), 53-99.

[3] T. Bartoszyński, H. Judah, Set theory. On the structure of the real line. A. K. Peters, Ltd., Wellesley, MA, 1995. xii+546 pp.

[4] T. Bartoszynski, H. Judah, S. Shelah, The Cichon diagram, J. Symbolic Logic, 58 (1993) 401-423.

[5] A. Blass, Combinatorial cardinal characteristics of the continuum in: Handbook of Set Theory (M. Foreman, A. Kanamori, and M. Magidor, eds.), Springer, 2010, pp. 395-491.

[6] S. Frick, S. Geschke, Basis theorems for continuous n-colorings, J. Combin. Theory Ser. A 118 (2011), 1334-1349.

[7] S. Geschke, Weak Borel chromatic numbers, MLQ Math. Log. Q. 57 (2011), 5-13.

[8] A. Kechris, Classical descriptive set theory, Springer-Verlag, New York, 1995.

[9] W. Kubis, Perfect cliques and $G_{\delta}$ colorings of Polish spaces, Proc. Amer. Math. Soc. 131 (2003), 619-623.

[10] L. Newelski, J. Pawlikowski, W. Seredyński, Infinite free sets for small measure set mappings, Proc. Amer. Math. Soc. 100 (1987), 335-339.

[11] S. Solecki, O. Spinas, Dominating and unbounded free sets, J. Symbolic Logic 64 (1999), 75-80.

[12] R. Solovay, A model of set-theory in which every set of reals is Lebesgue measurable, Ann. of Math. (2) 92 (1970) $1-56$.

[13] M. Talagrand, Filtres: Mesurabilité, rapidité, propriété de Baire forte, Studia Math. 74 (1982), $283-291$.

Department of Mathematics, Ivan Franko National University of Lviv, Ukraine; And

Instytut Matematyki, Jan Kochanowski University in Kielce, Poland.

E-mail address: t.o.banakh@gmail.com

URL: http://www.franko.lviv.ua/faculty/mechmat/Departments/Topology/bancv.html

Kurt Gödel Research Center for Mathematical Logic, University of Vienna, Währinger Strasse 25, A-1090 Wien, Austria.

E-mail address: lzdomsky@gmail.com

$U R L:$ http://www.logic.univie.ac.at/ lzdomsky/ 